\title{
The FIREBall-2 UV balloon telescope: 2018 flight and improvements for 2020
}

Hoadley, Keri, Hamden, Erika, Milliard, Bruno, Khan, Aafaque, Agarwal, Simran, et al.

Keri Hoadley, Erika T. Hamden, Bruno Milliard, Aafaque R. Khan, Simran Agarwal, Zeren Lin, David Schiminovich, Gillian Kyne, Jean Evrard, D. Christopher Martin, "The FIREBall-2 UV balloon telescope: 2018 flight and improvements for 2020," Proc. SPIE 11118, UV, X-Ray, and Gamma-Ray Space Instrumentation for Astronomy XXI, 1111815 (9 September 2019); doi: $10.1117 / 12.2530047$

Event: SPIE Optical Engineering + Applications, 2019, San Diego, California, United States 


\title{
The FIREBall-2 UV balloon telescope: 2018 flight and improvments for 2020
}

\author{
Keri Hoadley $^{\mathrm{a}}$, Erika T. Hamden ${ }^{\mathrm{b}}$, Bruno Milliard ${ }^{\mathrm{d}}$, Aafaque R. Khan ${ }^{\mathrm{b}}$, Simran Agarwal $^{\mathrm{b}}$, \\ Zeren Lin ${ }^{\mathrm{a}}$, David Schiminovich ${ }^{\mathrm{e}}$, Gillian Kyne ${ }^{\mathrm{c}}$, Jean Evrard ${ }^{\mathrm{f}}$, and D. Christopher Martin ${ }^{\mathrm{a}}$ \\ ${ }^{a}$ California Institute of Technology, Division of Physics, Math, and Astronomy, $1200 \mathrm{E}$ \\ California Blvd, MC 278-17, Pasadena, CA 91105, USA \\ ${ }^{\mathrm{b}}$ University of Arizona, Steward Observatory, 933 N Cherry Ave, Tucson, AZ 85721, USA \\ 'NASA's Jet Propulsion Laboratory, 4800 Oak Grove Drive, Pasadena, CA 91109, USA \\ dLaboratoire d'Astrophysique de Marseille, 38 Rue Frdric Joliot Curie, 13013 Marseille, France \\ ${ }^{\mathrm{e}}$ Columbia University, $550 \mathrm{~W}$ 120th St, New York, NY 10027, USA \\ ${ }^{\mathrm{f}}$ Centre national d'études spatiales, 18 Avenue Edouard Belin, 31400 Toulouse, France
}

\begin{abstract}
The Faint Intergalactic-medium Redshifted Emission Balloon (FIREBall-2, FB-2) is designed to discover and map faint UV emission from the circumgalactic medium around low redshift galaxies $(\mathrm{z} \sim 0.3(\mathrm{C} \mathrm{IV}) ; \mathrm{z} \sim 0.7$ $(\operatorname{Ly} \alpha) ; \mathrm{z} \sim 1.0(\mathrm{O} \mathrm{VI})$ ). FIREBall-2's first launch, on September 22nd 2018 out of Ft. Sumner, NM, was abruptly cut short due to a hole that developed in the balloon. FIREBall-2 was unable to observe above its minimum require altitude $(25 \mathrm{~km}$; nominal: $32 \mathrm{~km})$ for its shortest required time (2 hours; nominal: $8+$ hours). The shape of the deflated balloon, as well as a concurrent full moon close to our observed target field, revealed a severe, off-axis scattered light path directly to the UV science detector.

Additional damage to FB-2 added complications to the ongoing effort to prepare FB-2 for a quick re-floght. Upon landing, several mirrors in the optical chain, including the two large telescope mirrors, were damaged, resulting in chunks of material broken off the sides and reflecting surfaces. The magnifying optical element, called the focal corrector, was discovered to be misaligned beyond tolerance after the 2018 flight, with one of its two mirrors damaged from the landing impact. We describe the steps taken thus far to mitigate the damage to the optics, as well as procedures and results from the ongoing efforts to re-align the focal corrector and spectrograph optics. We report the throughput of the spectrograph before and after the 2018 flight and plans for improving it. Finally, we describe several methods by which we address the scattered light issues seen from FIREBall-2's 2018 campaign and present the current status of FB-2 to fly during the summer campaign in Palestine, TX in 2020 .
\end{abstract}

Keywords: Scientific Ballooning, CGM, Galaxy Evolution, Optical Alignment

\section{INTRODUCTION}

The Faint Intergalactic-medium Redshifted Emission Balloon (FIREBall-2, FB-2) is a joint NASA/CNES-funded suborbital astronomical balloon telescope designed to investigate science at the forefront of modern extra-galactic astronomy. FIREBall-2 benefits from improved, experimental technology that enables the next generations of space missions, including deformable mirror/grating surface figures, new detectors, and improved coatings for UV astrophysics. At the same time, the next generation of scientists and Principle Investigators are trained through FB-2. FIREBall-2's challenging design and forefront scientific investigations are complex enough to offer unique experiences in astronomical mission design and data analysis. Over the last decade, the FIREBall program has trained and supported over 10 graduate students to obtain their Ph.D.'s and has historically been lead by Postdoctoral Scholars in the astronomical field (most recently, the author of this proceeding).

Further author information: (Send correspondence to K. Hoadley)

K. Hoadley: E-mail: khoadley@caltech.edu

UV, X-Ray, and Gamma-Ray Space Instrumentation for Astronomy XXI, edited by Oswald H. Siegmund, Proc. of SPIE Vol. 11118, $1111815 \cdot$ ? 2019 SPIE · CCC code: 0277-786X/19/\$21 · doi: 10.1117/12.2530047 
This proceeding describes the compelling scientific case of FIREBall-2 and, briefly, its first flight in 2018. We describe the successes of the mission, as well as critical places for improvement before the next flight. Adsdition complications arose after FIREBall-2 was recovered after its flight, where key parts of the instrument were found severely damaged. We talk about efforts to mitigate this damage and the path forward to a flight of FIREBall-2 next year.

\subsection{The History \& Current State of Knowledge on IGM/CGM Physics}

The intergalactic medium (IGM) and circumgalactic medium (CGM) are fundamental aspects of our universe. Large-scale models of the $\Lambda \mathrm{CDM}$ universe predict sheets and filaments of dark matter arranged in a "cosmic web", where galaxies and clusters reside along and within over-densities and nodes in the web. Channeled along the web by gravity, baryons are expected to flow into galaxies to fuel star formation, and are later transformed and ejected by stellar winds, supernovae, and galactic activity. These "feedback" processes inject energy and metals into the galaxy halo and nearby IGM, but these overall processes are still poorly understood. Numerical simulations predict what properties we should expect from inflowing and outflowing material over a galactic histories, such as the size, angular momentum, and luminosity. ${ }^{1-5}$ However, without direct measurements of these properties, these theories are difficult to back up.

Until recently, directly observing the IGM/CGM to and its connection to the flow of baryons into and out of galaxies (which fuel and quench star formation, respectively), has been impossible to conduct due to their low luminosity. Absorption line studies of the Ly- $\alpha$ forest paved the way for indirect measures of IGM/CGM physics and validated the existance of the IGM/CGM. ${ }^{6-9}$ These observations, spearheaded by intensive surveys like COS-HALOS and COS-CCC, provided the first view of the make-up and physical state of matter found in the CGMs around nearby galaxies. Such surveys have conclusively detected absorption from HI, MgII, CIV, OVI and other ionized metals within the projected virial radius of foreground galaxies. These studies, however, are limited by their methodology: by nature, absorption spectroscopy detects gas through a single "pencil-beam" line of sight (LOS), where the absorption depth scales directly to the density. With this method, there is no way to distinguish between the presence of gas in filaments, a random collection of gas clumps, or a smooth distribution of gas within the CGM. Absorption line results, in turn, have motivated the push for direct emission detection from the IGM/CGM.

Since its discovery, the brightest components of high-redshift CGM gas have been directly measured using state-of-the-art ground-based instruments, like the Cosmic Web Imager on Palomar and Keck (PCWI/KCWI ${ }^{10-12}$ ) and Multi Unit Spectroscopic Explorer (MUSE ${ }^{13-15}$ ). Ground-based instrumentation are pioneering direct observations of IGM/CGM gas at high redshifts, where Ly- $\alpha$ is observable at visible wavelengths. Circum-quasar media (CQM) provide the easiest sources of detecting CGM in emission because the power from the quasar causes fluorescence in the CGM gas, making it more luminous. Most studies have taken advantage of this boosted emission and have discovered important properties about the CQM at early cosmic times. High-redshift $(\mathrm{z}>3)$ surveys have found a $100 \%$ incidence of large Ly- $\alpha$ nebula around QSOs, ${ }^{13,15}$ while at slightly lower redshifts $(2.2<\mathrm{z}<2.8)$, CQM surveys find less incidence of large Ly- $\alpha$ structures, perhaps pointing to a key evolutionary change over cosmic time. ${ }^{16}$ Spatially- and cosmically-close QSO pairs have been found to harbor extremely large Ly- $\alpha$ nebulae, linking cosmic over-densities to these structure. ${ }^{17}$ Some large Ly- $\alpha$ nebulae have been inferred to have complex velocity structures, providing tantalizing evidence of bulk gas rotation and radial inflows. ${ }^{12}$ Tentative detections of CGM emission from non-QSO galaxies have also been inferred in recent years. There is evidence for CGM emission from the halos of Lyman Break Galaxies between redshifts of $\mathrm{z} \sim 2-3{ }^{18,19}$ Filamentary extensions between Lyman Alpha Blobs, particularly in the system SSA22, have been observed and show spectral properties consistent with inflowing gas from the cosmic web. ${ }^{11}$ Extended Ly- $\alpha$ emission has been seen around star forming galaxies, interpreted as resonance scattering within their CGMs. ${ }^{20}$ Insights into the CGM and its connections with IGM structures for non-QSO galaxies is particularly important for understanding the formation and evolution of normal, Milky Way-like galaxies, linking the diversity of galaxies (from star-bursting galaxies to "red \& dead") we observe in the universe presently with their histories.

\subsection{FIREBall Science Goals \& Instrument Description}

FIREBall-2's main science objectives are to discover and map the low-redshift CGM/CQM around low-redshift galaxies. FIREBall-2 accomplishes this by taking advantage of atmospheric $\mathrm{O}_{2}$ and $\mathrm{O}_{3}$ absorption band gaps 


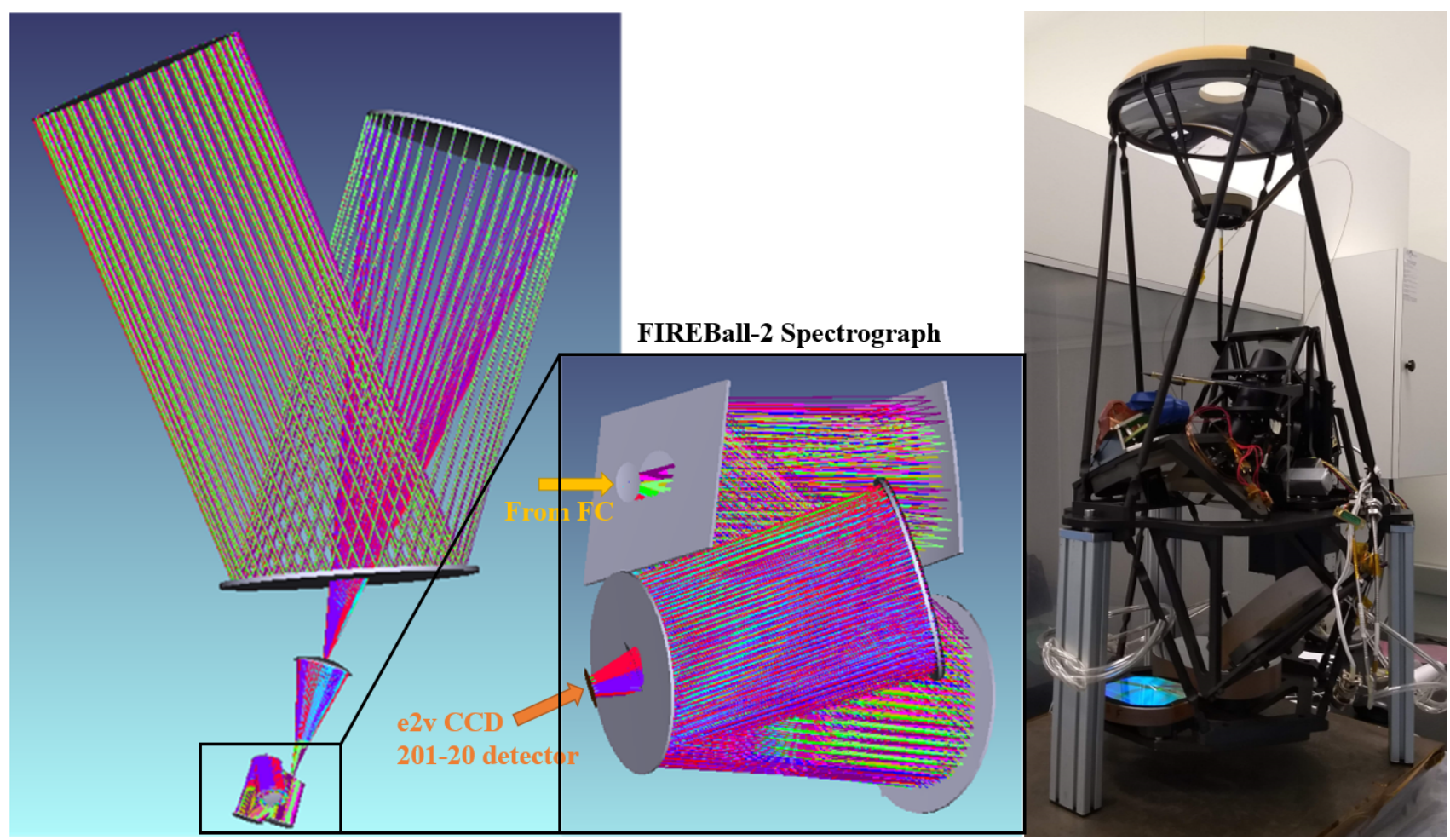

Figure 1. Left: The FIREBall-2 light path through the entire instrument assembly. A zoomed-in view of the spectrograph raytrace is shown, including where the telescope light enters the spectrograph ("From FC") and the UV detector. Right: The FIREBall-2 spectrograph as-built, out of its vacuum vessel and awaiting further testing after the 2018 flight out of Ft. Sumner, NM.

at scientific balloon float altitudes $(>30 \mathrm{~km}),{ }^{21}$ where it observes strong UV emission lines (Ly $\alpha$ (z 0.7); OVI $(\mathrm{z} \sim 1.0)$; and $\left.\mathrm{CIV}(\mathrm{z} \sim 0.3)^{*}\right)$ in the CGM/CQM of targeted galaxies. FIREBall-2 takes a statistical survey of CGM emission and gas properties, which provides important constraints on cosmological models of how gas is transported to and ejected from galaxies as they evolve, particularly closer to the present state of the universe.

The FIREBall-2 design is a near-UV ( $\lambda=2000-2100 \AA)$ multi-object spectrograph (MOS). FIREBall-2 provides a complimentary facility to cutting-edge ground-based instrumentation, designed to study the strongest gas tracers in the CGM around hundreds of low redshift galaxies and QSOs over the duration of one flight ${ }^{\dagger}$, a unique capability in the current landscape of IGM/CGM astrophysics. FIREBall-2 is an upgrade to FIREBall-1: ${ }^{22}$ FIREBall-2 has an increased field of view (FOV), more targets available to observe simultaneously via predefined and custom-made field slit masks, and has improved efficiency by taking advantage of state-of-the-art UV technology. FIREBall-2 flies with a 1-meter diameter primary mirror inherited from FIREBall-1, which directs light to a field corrector (FC). The FC focuses and magnifies the FOV to give FIREBall-2 its wide field and access to many galaxies simultaneously. At the focus of the FC is a slit mask wheel, which includes a selection of custom-designed sky masks for targeted CGM observations and calibration masks to focus and calibrate the spectrograph. The mask wheel has multiple purposes: each mask is custom-made to match the shapes of the focal plane of the FC, so that focused images go through the carefully-determined slit positions etched into the mask, and provides focused light to the spectrograph; each mask is also reflective and directs the image plane not transmitted through the mask slits to a guiding system. The guider camera at this point in the optical chain is critical for verifying focus at the UV detector and provides guidance using bright (V-mag $>12)$ field stars during flight. More information on the guider system and its performance can be found in an upcoming publication. ${ }^{23}$

The spectrograph consists of two flat mirrors, two Schmidt mirrors, a novel diffraction grating, and a UV-

*OVI (1035), Ly $\alpha$ (1216), and CIV (1548) are all redshifted to $\lambda=2000-2010 \AA$ at these redshifts

${ }^{\dagger}$ One flight is $\sim 24$ hours, or one observing night, which gives FB-2 $\sim 8$ hours of observing time 
optimized electron-multiplying (EM)CCD. Focused galaxy/CGM images passing through the mask slits to the first flat mirror, where the light is directed to the first Schmidt mirror. Light is diffracted off the grating, where $1^{\text {st }}$-order near-UV photons go to the second flat mirror, the second Schmidt mirror, and finally reach a focus on the UV EMCCD. Figure 1 shows a ray-trace of FB-2 and the as-built FC and spectrograph.

The FB-2 grating is an anamorphically-aspherized reflective Schmidt grating, which corrects for spherical aberrations in the FC image quality. It has a line density of 2400 lines $/ \mathrm{mm}$ and angle of incidence of $28^{\circ} .{ }^{24}$ The FB-2 UV EMCCD is provided by the Microdevices Laboratory (MDL) at JPL (PI: S. Nikzad ${ }^{25-27}$ ) as a part of the FB-2 collaboration. A Teledyne e2v CCD201-20 wafer is thinned and $\delta$-doped using a molecular-beam epitaxy, which grows a single layer of boron-doped silicon. ${ }^{26}$ This process significantly increases the quantum efficiency (QE) of the device. An AR coating is added at the end of the process to overcome QE limitations due to the reflectivity of the silicon. A 3-layer AR coated was optimized for the FB-2 bandpass, which gave a peak $\mathrm{QE} \sim 65 \%$ and $\mathrm{QE}>50 \%$ across the bandpass. ${ }^{25,27}$
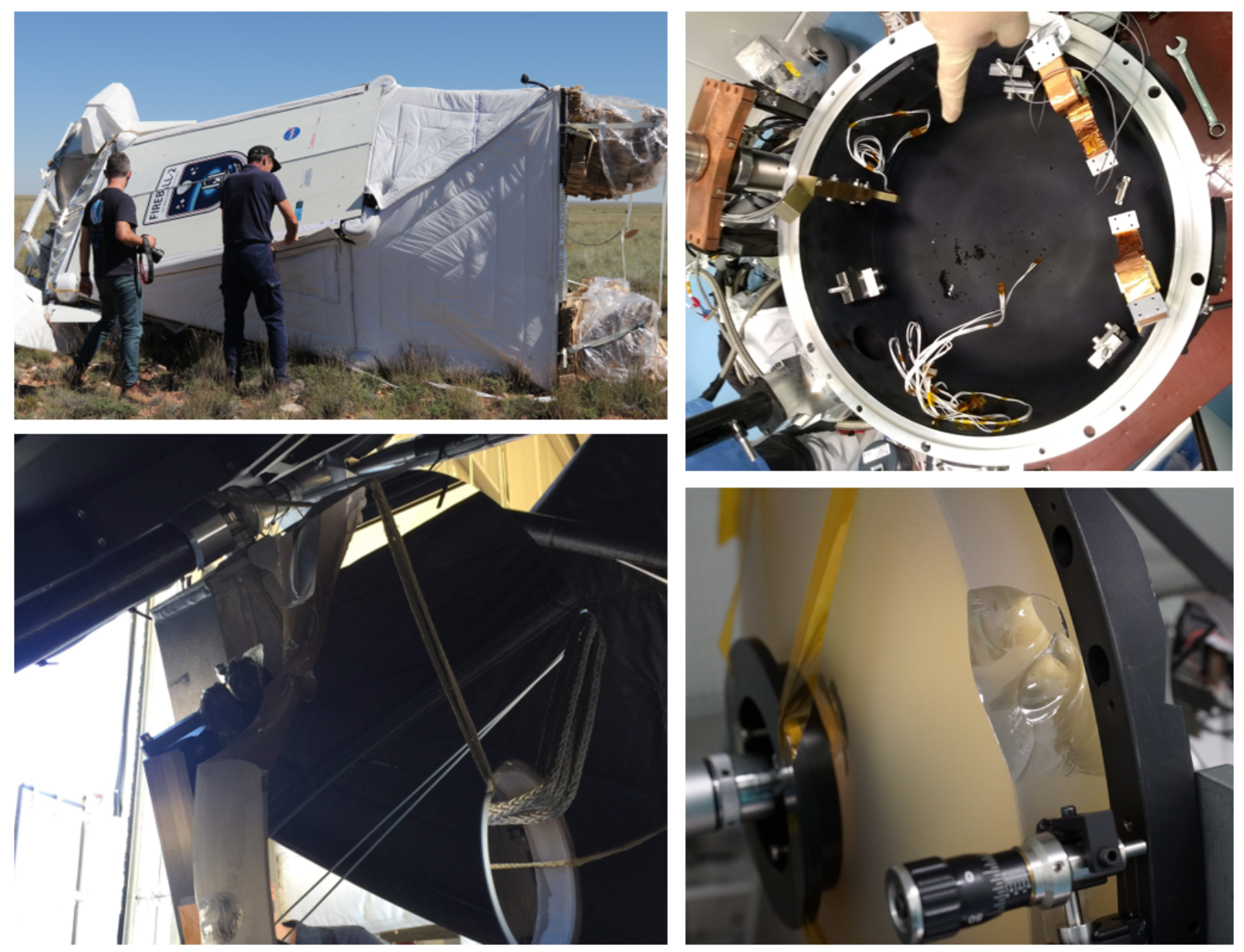

Figure 2. Top, Left: The recovery team found FB-2 on one side the night after the flight. Bottom, Left: Both large telescope mirrors took significant damage from the landing of $\mathrm{FB}-2$, with $\sim 5 \%$ loss in collecting area of the primary mirror. Top, Right: Upon opening the spectrograph vacuum tank, the team discovered that charcoal from the getter spilled all over the optics and tank, leading to a significant contamination control effort. Bottom, Right: The large FC mirror also broke at one bond pad location, leading to a clam-shell fracture at the location of this mount and mis-aligning the optical path out of allowed tolerances. 


\section{THE FIREBALL-2 2018 CAMPAIGN}

FIREBall-2 flew in the fall of 2018 from Fort Sumner, NM. The release and ascent of the payload went smoothly, with no clear signs of damage to either the balloon or instrument. Shortly after float altitude was reached, it was found that the balloon had developed a puncture hole and was slowly leaking helium. As the day transitioned to night, the fall in altitude due to changing atmospheric conditions exacerbated the altitude lost by the balloon leak and FIREBall-2 fell at a quicker rate. After initial calibrations and acquisition on the first science field, FIREBall-2 was only able to observe one of its five science targets (four large survey fields and one MgII-absorbing QSO) for half the time needed to build enough signal to detect CGM photons above the minimum float altitude required to access the NUV 2000 - $2010 \AA$ atmospheric window $(>25 \mathrm{~km})$. At around 1AM MST, FIREBall-2 was released from the balloon and fell back to the ground with a parachute. A recovery team located the payload and returned it to Fort Sumner later in the day.

\subsection{State of the Instrument after 2018 Flight}

Upon initial inspection, it was clear that FIREBall-2 took a hard landing. The payload was found on one side (Figure 2), and damage to the gondola structure, primarily to the carbon fiber struts along one side of the mechanical structure, was observed. Upon return to the CSBF Flight Facility in Fort Sumner and closer inspection of the inside of the gondola, damage to both large telescope mirrors was seen. The flat siderostat and parabolic mirror had broken off chunks of glass along one of three bond pad locations (Figure 2). Once the payload was shipped back to France and the spectrograph could be examined more closely, it was found that the cryocooler, which cooled the UV EMCCD to operating temperatures, was broken, likely due to the impact from landing. Opening the spectrograph, charcoal from the getter was found everywhere in the tank, coating the mirrors and detector and the opto-mechnaical structure. One of the focal corrector mirrors, FC2, developed a clamshell fracture in the optic on one of its three bond pads (Figure 2).

Overall, the payload as a whole remained intact after the 2018 flight. The spectrograph remained mostly aligned, with the broken focal corrector mirror then main culprit of misalignment. Post-flight calibrations and scattered light tests were able to be performed with little-to-no adjustment to the spectrograph (except a replacement of the cryocooler and vacuum charcoal getter).

\subsection{Flight successes}

Overall, the FB-2 payload worked very well during its short flight. Initial UV detector images were take during twilight to calibrate the Gain settings for the EMCCD. Images of varying exposure times were taken to balance the cosmic rate rate seen at float altitudes to the image area lost to these impacts. Guider and gondola rotation algorithms were tested and verified before acquiring the first science field. Once astronomical night began, the instrument found its science field successfully and guided on the field for long-duration observations for $\sim 1$ hour, despite the altitude loss from the leaking balloon.

FIREBall-2 demonstrated that its complex target acquisition and guiding algorithms kept the instrument aligned with the target fields to a better pointing accuracy than required (Requirement: $1^{\prime \prime}$ RMS over 1 hour; demonstrated 0.4" RMS over 1 hour ${ }^{28}$ ). The UV EMCCD worked in Gain-mode (for photon-counting) for the duration of the flight, providing a critical space qualification test for UV EMCCDs and the $\delta$-doping/AR coating processes being developed by JPL-MDL. The in-flight thermal system, set up to ensure the EMCCD and guider cameras were sufficiently cool to run, kept the system to within operating temepratures for the duration of the mission.

\subsection{Lessons Learned from the Flight \& Campaign}

While FIREBall-2 demonstrated many successes over the flight, there were a few hiccups that, if the mission had gone on for its intended length, would have lead to significant issues and potential failures. The most concerning of these issues was a large scattered light leak. FIREBall-2 flew during a nearly full moon, and coupled with the deflated balloon shape, created a scattered light background $\sim 100 \times$ higher than our expected background level (Figure 3, left). At certain orientations, moonlight was focused directly through the system, which led to "flashes" that were still many times higher in flux than our expected background and CGM signal (Figure 3, right). "Flashes" images had to be completely discarded from future analysis, as the background signal was too strong and spread over the entire image to recover any significant signal. 
Towards the end of our observations on the first science field, the gondola rotation stage failed. The rotation stage physically rotates the spectrograph while FB-2 observes a target, ensuring that the guiding-to-science slits orientation and focus remains the same during the science exposures. Without it, the spectrograph cannot track with the night sky movements over the course of the observations. While this failure did not impact the observations taken on the first science field, it would have lead to an inability to track the targets in other science fields.

Finally, several critical elements in the thermal stability chain failed at the beginning of the observations. The EMCCD cooler/heater system did not work, though fortunately the CCD remained at the desired temperature $\left(-110^{\circ} \mathrm{C}\right)$ during the flight. The external cooling system also stopped circulating. Both failures would have led to overheating of both the detector and critical cooling elements in the payload, to the point of damage to these mechanisms and elevated dark current rates in detector images.

\subsection{Action Items for Future Flights}

Before the next launch of FIREBall-2, all of these points of failure discovered during the first flight of FIREBall-2 must be investigated and addressed. In order of priority, we have determined that:

1. Additional stray light mitigation is required;

2. All damaged and broken elements of the instrument must be addressed, including the gondola structure, gondola rotation stage, and FIREBall-2 telescope mirrors;

3. A re-examination of the instrument performance and alignment of optical elements is needed.

In addition to required action items, there are several desired changes to the instrument to alleviate noise sources from the detector and mainstream the installation and use of complicated aspects of the system. Some of these changes include: re-building the on-board detector computer to handle capturing, recording, and down-linking both detector images and housekeeping data in real-time during flight; upgrading the current CCD device for one with lower cosmetics (e.g., hot pixels) and with a visible light-blocking coating (the current one has comparable NUV quantum efficiency (QE) and visible light QE); upgrading the EMCCD controller from NUVU v2 to NUVU v3 (for better control on read-out noise); and re-designing the cold chain from the cryocooler to the detector for more efficient heat transfer.

\section{THE ROAD TO FIREBALL-2020}

There are a number of critical action items and hardware improvements, both required and desired, that have to happen for FIREBall-2 to launch launch out of the Columbia Scientific Balloon Facility in Palestine, TX during the next campaign season in 2020. Below, we discuss the status of different actions since the flight of 2018, with the goal of being ready for a re-flight in early 2020. 


\subsection{Large Mirror Refurbishments}

In January 2019, the optician who ground the FB-1 telescope mirrors, James Mulherin, inspected the damage taken by both the primary mirror and siderostat aft the 2018 flight. He found no propagating cracks or signs of stress through the optics, and recommended that we have the damaged surfaces machined and smoothed to avoid further damage. During the spring, both optics were shipped to Goddard Space Flight Center to be machined, stripped of their $\mathrm{Al}+\mathrm{MgF}_{2}$ coatings, and re-coated over the course of the summer. Both large optics have been machined and smoothed where damage was taken. The primary mirror has been stripped of its coating off-site and is ready to be re-coated in Goddard's large evaporation chamber. The siderostat is scheduled to be stripped of its previous coating at the beginning of August. A recent development with the coating chamber, which resulted in the power supply for the deposition being replaced, set back the schedule for re-coating by a few weeks, but the coating runs are still likely to happen before September. Currently, the large optics critical path is on-schedule to meet the re-alignment in the CNES gondola before the end of the year.

\subsection{CNES Gondola Improvements and Baffling Plans}

CNES has successfully replaced all damaged carbon fiber struts from the landing with spares at their Toulouse facility. The gondola floor was also repaired. The culprit of the rotation stage failure was found to be inadequately short screws being used to hold the elastic ring in place of the axle mechanism. The rotation gears were found loose and full of grease after the FIREBall-2 flight. A joint effort by CNES and LAM is in place to refurbish these axle mechanisms and use longer screws to ensure that enough threads are in placed to keep the mechanism from failing again.

CNES is helping with the scattered light mitigation by blackening what they can within the gondola itself deter glancing reflections through the payload and into the spectrograph. They have blackened all metal/reflective surfaced within the gondola and on the telescope doors. In addition, two gondola baffles are being explored to avoid significant light leaks from the Moon, in the chance that FIREBall2 must fly during a full moon or with the moon within a given angular distance to our science targets: a "tent", or a fold-out baffle that blocks light coming directly above the instrument into the spectrograph, is one option, and the other is a "cornet" in the siderostat central hole that directs only onaxis light into the spectrograph tank window.

\subsection{Spectrograph Improvements}

Several critical paths must be addressed with the FIREBall-2 spectrograph before a re-flight of the payload is possible. The FIREBall-2 spectrograph is highly complex, with moving parts during flight and tight tolerances to ensure both focused UV spectra and guide stars for the fine-pointing system. A detailed description of the spectrograph and the relationship between the guiding system and spectrograph is found in other literature. ${ }^{23,24}$ We breakdown the work needed to address key spectrograph issues and improvements below.

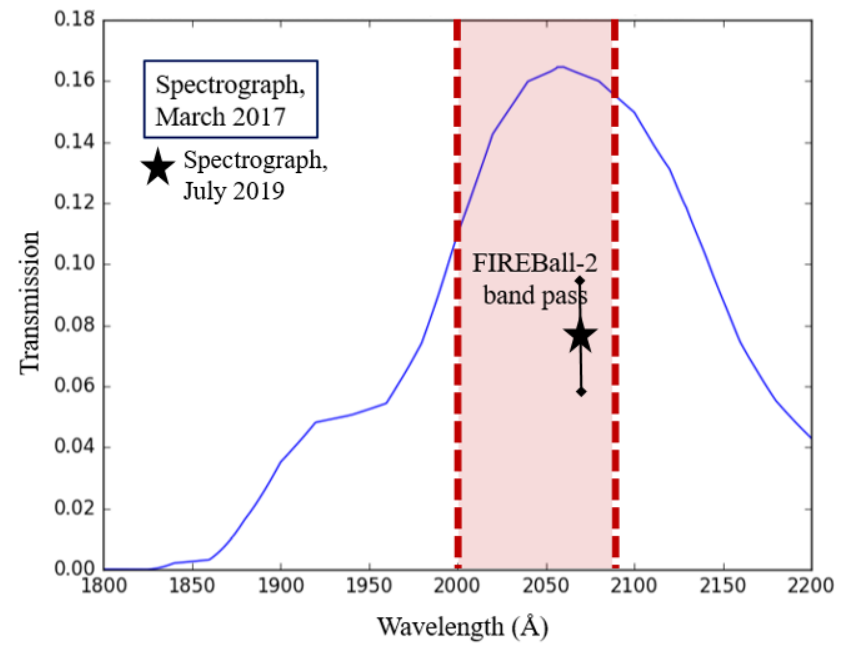

Figure 4. The throughput (transmission) measurement of the FIREBall-2 spectrograph before its planned 2017 campaign (blue line) and the measurement taken after the 2018 flight, before re-build for the 2020 campaign (black star with errors). The FIREBall-2 narrow bandpass is shaded red. The throughput is measured as the total throughput of the entire spectrograph and FC assembly, which includes: two FC mirrors, a slit mask, two flat mirror, two Schmidt spherical mirrors, and one $1^{\text {st }}$-order diffraction grating. All mirrors are coated with $\mathrm{Al}+\mathrm{MgF}_{2}$ by Acton Optics, which have $\sim 90 \%$ reflectance in the FIREBall-2 bandpass. The grating is a bare-Al coating with no protective overcoat. We are exploring reasons why the throughput has degraded since 2017, including oxidation of the grating coating and a possibility of a contamination in the spectrograph. 


\subsubsection{Post-flight Throughput measurements}

FIREBall-2 is a cutting-edge facility that will observe some of the faintest signals in the low-redshift universe. To accomplish this, FIREBall-2 requires exquisite sensitivity and low background noise during the entire observing period of any given target. Therefore, ensuring that the throughput of the optical system remains the same as measured before the 2018 flight is critical to verify that the instrument will capture the faint signal from the CGM around our chosen galaxies.

Figure 4 shows the throughput of the focal corrector mirrors and the spectrograph optics, without the UV CCD QE folded in to the measurement. The throughput was first measured in Spring 2017, when all the optics were first aligned. Within the FIREBall-2 bandpass of interested (highlighted in the red region), the throughput is measured to be $>10 \%$ across the band, peaking at $\sim 17 \%$ at $2060 \AA$. After post-flight calibrations, the spectrograph was removed from its vacuum vessel to address several hardware and contamination issues that arose from FIREBall-2's landing. While the spectrograph bench was out, the throughput of the optics was re-measured. Figure 4 shows the one measurement made of the FIREBall-2 spectrograph and focal corrector throughput at $2060 \AA$ (black star symbol). The throughput is now measured at $\sim 7 \%$, roughly a $2.5 \times$ loss in sensitivity at the peak of our throughput.

In light of this significant loss in sensitivity, the team has begun investigating possible culprits that could cause this loss. Two main routes have been examined so far: the grating, which is a bare aluminum coating without a protective over-coating, such as $\mathrm{MgF}_{2}$ (which all the other optics have), and possible contamination from particulates and/or long-chain molecules, which could be attributed to the charcoal dust spill from the vacuum getter discovered after FIREBall-2's recovery.

We have a flight spare of the grating, which the team measured the relative performance of during the first throughput measurement. It was found that the flight grating (bare Al coating) had $10 \%$ better efficiency than

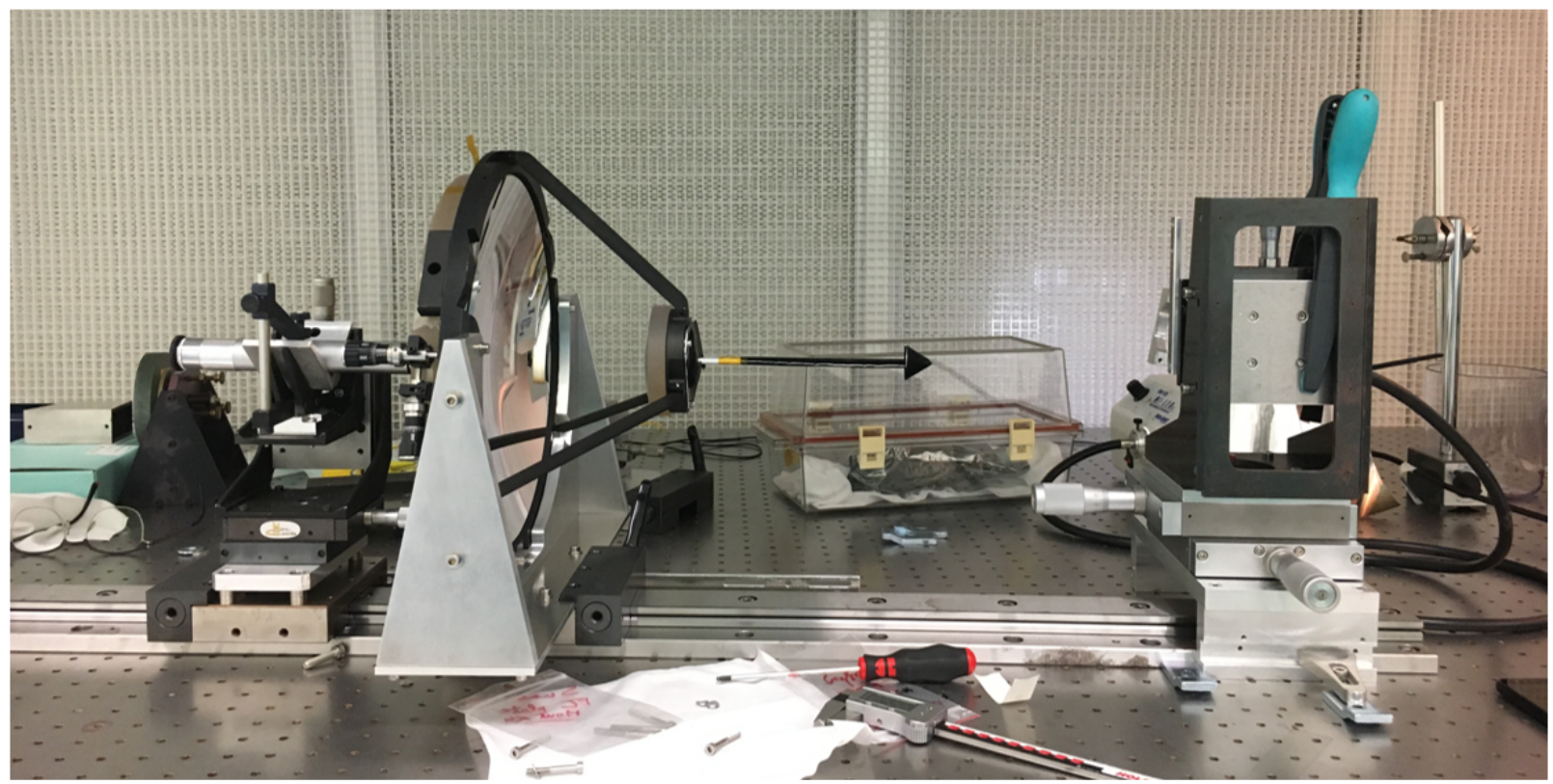

Figure 5. A picture of the FC mirrors attached to their alignment optical bench (the "horizontal bench") in a clean room at LAM, as of August 2019. After the 2018 flight, it was discovered upon opening the spectrograph tank that the large FC mirror, FC2, had a clam-shell fracture in the mirror at the location of one of its bond pads. After measuring and comparing the post-flight alignment of the FC mirrors with pre-flight aliment measurements, it was found that the FC assembly was way out of alignment tolerances, which have to be addressed. FC2 is the only mirror we will move to realign the system, at the location of the broken bond pad. At this position on FC2, an "adjustment tool', which moves the mirror in its rotation axis and towards/away FC1, has been attached to the mirror. We will use the procedure developed during the first alignment of the FC mirrors to re-align the system, using only this tool. 
the spare (coated with $\mathrm{Al}+\mathrm{MgF}_{2}$ ). During the latest throughput measurements, the spare was swapped in after the throughput measurement with the flight grating, and it was found that the relative throughput measurement increased by $20 \%$ relative to throughput with the flight grating. It is clear that the bare-Al coating has degraded somewhat, but not enough to account for the total throughput lost in the entire spectrograph. An additional $\sim 5 \%$ loss in efficiency from all the optics must be accounted for to make up for the total throughput lost over all the optics in the spectrograph and focal corrector.

Since the charcoal contamination was discovered, it seems entirely plausible that the main culprit for the rest of the sensitivity degradation is fine dust particles coating the optics. The spectrograph was shipped from Ft. Sumner, NM to Toulouse, France, and then underwent several vacuum cycles during the time we had the charcoal contamination. These actions could have moved particles around the tank, where they stuck to many different surfaces. Since the optical surfaces present large surface areas for particles to stick to, it is likely they have contaminated these reflective areas.

Currently, each optic has been uninstalled from the spectrograph bench and is undergoing a thorough cleaning. The cleaning process has been adapted by best-practices methods provided by Goddard Space Flight Center and Acton Optics. The flight grating, given it is a bare-Al coating, will not undergo this cleaning process, and will only be blown off for particulates. After each optic is cleaned, they will be re-installed onto the spectrograph bench with adjusted shims for nominal instrument performance. The throughput of the entire optical path will be re-measured after alignments are complete, to verify that the cleaning process worked as intended. If the cleaning of each optic worked and accounts for the rest of the loss in sensitivity (without the grating), the expected spectrograph and focal corrector throughput at $2060 \AA$ is $\sim 13.5 \%$.

\subsubsection{Focal Corrector Adjustments}

After it was discovered that one of the focal corrector mirrors (FC2) had broken along one of its bond pads, we immediately planned to re-align the system. The focal corrector provides the necessary image quality corrections over the wide field fed to it by the primary mirror $\left(11^{\prime} \times 35^{\prime}\right)$, to ensure that all target spectra meet the instrument spatial resolution requirements (PSF $<=4^{\prime \prime}$, to successfully disentangle galactic emission from CGM gas emission). Because we require a small spatial resolution over a large field, the tolerance of the focal corrector mirrors is tight $-<200 \mu \mathrm{m}$ decenter and shift between them and relative to the slit mask plane.

A horizontal bench has been designed to align the focal corrector during the first build of FIREBall-2. Figure 5 shows the focal corrector set up on this horizontal bench, as of early August 2019. Adjustments in the alignment between the mirrors is ongoing and scheduled to be complete before September. To align the mirrors, a $5 \mu \mathrm{m}$ pinhole is set up where the center of the science masks is located relative to the back of FC1. An LED is fed through the pinhole to intercept first $\mathrm{FC} 2$, which reflect light to FC1, and an imaging $\mathrm{CCD}$ is set up in the aperture hole of $\mathrm{FC} 2$, along with a reticle to track the center and image quality of the measured spot. Once the distance between the pinhole and back of FC1 is set to within the allowed tolerance, an adjustment tool, which moves FC2 at the broken bond pad region in rotation and tip/tilt (Figure 2, bottom right), is used as the only means to adjust the image size, quality, and centering in the reticle image. Once the spot size reach a minimum with acceptable aberration qualities, a new bond pad will be epoxied between FC2 and its corona (mount). Once cured, we will very that the spot size and image quality remain the same or within a tolerable change before dismounting the adjustment tool and re-aligning the focal corrector to the spectrograph.

\subsubsection{Scattered Light: Identifying Stray Light Paths, Solutions, \& Implementation}

After the 2018 flight, the spectrograph underwent a series of post-flight calibrations, primarily to identify the source and preferential orientation of light entering the spectrograph that causes the severe light contamination seen during flight. Figure 6 shows the scattered light profile during flight (left) and the scattered light produced during post-flight calibrations in January (right). The scattered light image shape and brightness was relatively reproduced when light entered the spectrograph tank lens at a shallow angle and a particular orientation, which matched well with the orientation of the spectrograph during flight for the CGM Field (F2) observed.

Zemax simulations of this stray light path have been investigated, and we identified a direct light path from the top of the tank lens to the UV detector (which is also sensitive to visible light). Figure 7 shows how the light 


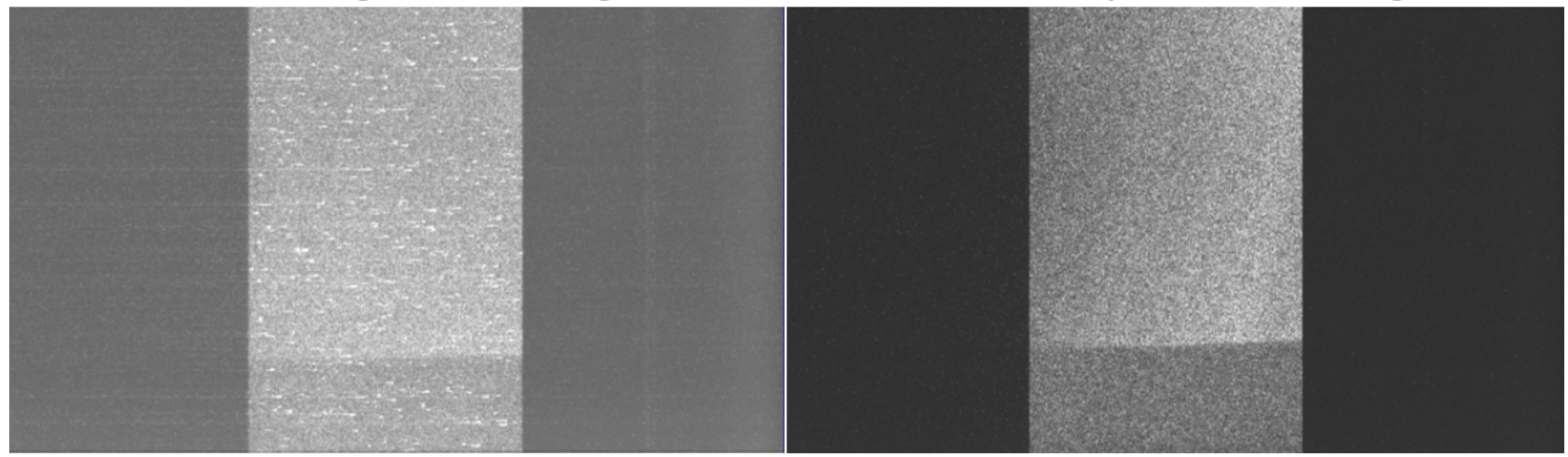

Figure 6. Left: During flight, FIREBall-2 saw a higher-than-expected scattered light background, primarily due to the deflated balloon profile funneling in moonlight for the duration of the flight. Right: Post-flight calibrations focused heavily on reproducing the light leak observed during the 2018 flight. In certain orientations that visible light enters the spectrograph, we have been able to reproduce the scattered light profile seen during flight.

entering the system can reach the back surface of the CCD. To address this direct path, a planar baffle, which already exists on this plane, will be extended to block incoming, off-axis light from reaching the UV detector.

Other avenues where light could scatter off several surfaces before intercepting the detector have been identified. One path is visible light diffracted off the grating, which is directed to a grazing incidence reflection off the side of the vacuum tank. While the tank itself is anodized black, a grazing reflection is more efficient than a shallow reflection, and we expect this grazing reflection, which directs light up to the UV detector, to be another source of stray light background. We will add levels of baffles to create a light trap near the grating.

Finally, during flight, it was observed that with the spectrograph tank shutter closed, a significant light leak was observed. While no such light leak was seen from testing the integrity of the shutter on the ground, it appears plausible that the main source of this light is Cherenkov radiation. Cherenkov radiation is produced when high-energy particles interact with materials of a different index of refraction than the air (or vacuum) the are in, and produce a spectrum of NUV-visible light. FIREBall-2 has a lot of optics that have uncoated areas for high-energy particles to interact with. We will mitigate Cherenkov radiation by blackening all the unused optical surfaces that could source Cherenkov radiation, with the intention that if Cherenkov radiation is produced within the optics, that the black material will prevent the light from leaking into the spectrograph. In addition, more blackened surfaces will help baffle from other currently undiscovered stray light routes through the spectrograph.

\subsection{Improvements to Other FIREBall-2 Systems}

On-board Shared Network for Housekeeping: During the last flight, we witnessed significant strain on the flight computers, particularly the science detector computer, due to both age of the hardware and OS systems, as well as the multiple routines and tasks they were set up to handle. In loo of requiring critical flight computer systems to handle secondary tasks to their designed purposes, like collecting and down-linking housekeeping data of the spectrograph and cooling systems, we are designing a dedicated on-board network that will route all temperature sensors to a common hub. This hub, designed to handle serial signals from on-board sensors and down-link through an Ethernet port for fast transmission over a CSBF-provided 8 (or 12) MB transmitter, alleviates the load put on critical flight computers handling the acquisition of science data, storage of that data, and transmission of data to the ground. We have ordered the necessary hardware for this network, and LAM are leading the effort to route all flight Arduino boards (which capture the sensor signals) through this network and to a dedicated transmission line.

Simplifying the Cooling System: Currently, the on-board cooling system, which provides a cold water chain to hardware in the spectrograph that requires heat exchange (e.g, the detector controller, the cryocooler 
reject, and the guiding camera system), is complicated and requires potential contamination issues to the spectrograph because it requires thermal grease at some contacts near spectrograph tank ports. Both the cryocooler and the newest version of our detector controller, Nuvu v3, come with vendor-installed radiators that have water-cooling ports for such a chiller system as is used on FIREBall-2. We have purchased both radiators and will replace them with the thermal grease-required chiller chain along the spectrograph to remove this source of contamination from the system and allow for easier installation of these components in the cooling chain.

Upgrading the Detector Controller \& Detector Flight Computer: FIREBall's UV EMCCD requires a Nuvu controller for photon-counting capabilities. For the 2018 flight, the Nuvu v2 controller was used and optimized for the mission. While it worked well, we were right on the noise limits of the Santec cable required of the controller to the detector board. For upcoming flights, we are moving towards integrating the new model of Nuvu, Nuvu v3, which is the current planned EMCCD controller for WFIRST. The controller comes within its own pressure vessel, which the v2 did not, and has the option for a radiator jacket that includes an inlet for water cooling. We have ordered the new controller with the radiator jacket and are testing it on the ground. The new controller software requires a more up-to-date OS system to run, so the detector flight computer is being completely upgraded. The previous flight detector computer will be kept as it is to run the Nuvu v2 in case the new flight computer and/or controller are not ready for integration into FIREBall-2 by the time we are ready to build. The new flight computer will have a custom-built pressure vessel, separate from the previous computer. The only component between the two computers that will be swapped is the cryocooler controller, which is housed inside the detector pressure vessel alongside the computer itself. The pressure vessel and flange, with connectors sealed to the flange, are in the process of being designed and machined.

\section{FIREBALL-2020 \& BEYOND}

FIREBall-2 is currently on-track to launch out of Palestine, TX

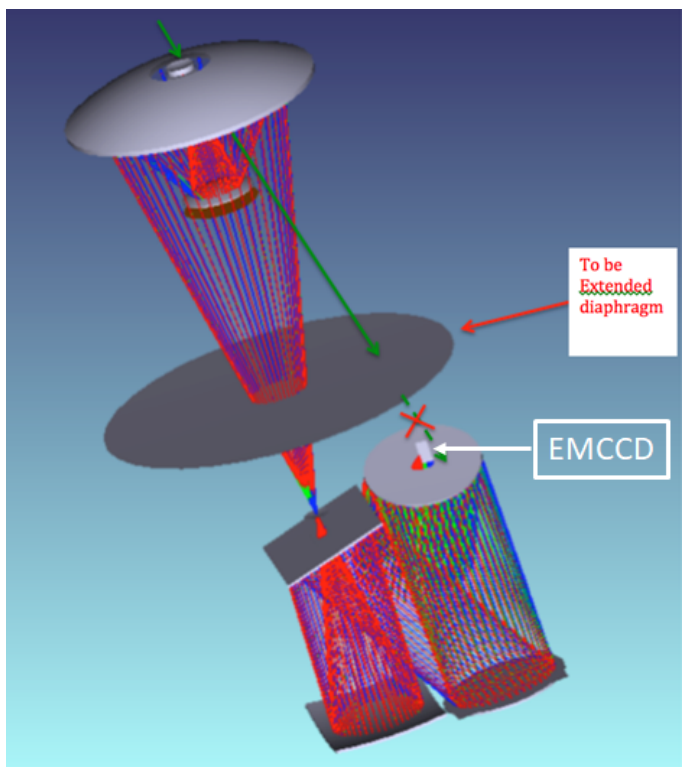

Figure 7. We now have a good understanding of the stray light path causing the high background count rate during flight. One of the primary paths was found to be off-axis light entering the spectrograph and having a direct line of sight to the back of the UV detector. Currently, there is a baffle in place between the FC mirrors and the slit masks to stop down the pupil image, and a simple extension of this baffle to cover the light path to the detector can prevent this direct sightline between off-axis light and the detector. in the Summer of 2020. All critical hardware adjustments are being addressed and are on-schedule to meet instrument integration and testing operations, starting in October 2019. All spectrograph operations are happening at LAM in Marseille, France. Spectrograph I/T will begin in October, where the spectrograph will be installed in its vacuum vessel, the slit mask carousel will be installed and aligned, the UV EMCDD will be installed and focused, and all spectrograph-related testing and calibrations will occur. Concurrent to spectrograph I/T, the large optics will arrive at CNES in Toulouse, France by midOctober. The optics will be aligned in the gondola at CNES before the end of the year. By December 2019, the spectrograph will be delivered to CNES for full end-to-end integration of FIREBall-2 at CNES. The team will perform a series of experiments and test to calibrate and understand the performance of the system, in particular making sure that the pointing, guiding, and UV focus mechanisms work together as they will during flight. Important tests on the cooling system, vacuum systems, and telemetry will also occur. By the beginning of March 2020, full integration of FIREBall-2 will be complete, and the entire gondola will be shipped to Palestine, TX for flight readiness.

A new launch site for FIREBall-2020 requires new targets and custom slit masks to observe CGM field available from Palestine in the summer. Columbia University, along with LAM, are spearheading this effort to identify fields of interest from existing galaxy surveys. We have identified two CGM fields of interest, including one observed during FIREBAll-1 (the GROTH strip), and two QSO fields - one with 5 QSOs and one QSO-pair 
at redshifts between $z=0.65-0.7$ and one with a very closely-spaced QSO-pair and a handful of Ly $\alpha$ CGM galaxies. Target masks will be cut at the University of Arizona in early September.

FIREBall-2 has another launch slated for the Fall of 2022 out of Ft. Sumner, NM. This launch will see the use of Nuvu v3, if it is not integrated into FIREBall-2020. We will also test a new red-blocking AR coating on the UV CCD, which should help alleviate many of the scattered light issues we witnessed during the 2018 flight ${ }^{\ddagger}$. These filters are currently being developed at JPL (Lead: S. Nikzad), in particular to be flown on a future FUV/NUV CubeSat mission, SPARCS. ${ }^{29}$ We will also work on developing a more narrow-band, 5-layer AR coating for a flight $\delta$-doped UV CCD, which will provide higher QE in the narrow bandpass critical for FIREBall-2 science.

\section{ACKNOWLEDGMENTS}

This work was funded primarily through the APRA program for sub-orbital missions which funds the US side of FIREBall. CNES and CNRS provided support for the French side of the FIREBall collaboration. Dr. Hoadley is supported through the David \& Ellen Lee Prize Postdoctoral Fellowship in Experimental Physics at Caltech. The FIREBall-2 collaboration would like thank the Columbia Scientific Ballooning Facility (CSBF) and Balloon Program Office (BPO/NASA) for their support throughout the 2017/2018 campaigned in Ft. Sumner, NM.

\section{REFERENCES}

[1] Cen, R. and Ostriker, J. P., "Where Are the Baryons? II. Feedback Effects," ApJ 650, 560-572 (Oct. 2006).

[2] Bertone, S., Schaye, J., Booth, C. M., Dalla Vecchia, C., Theuns, T., and Wiersma, R. P. C., "Metal-line emission from the warm-hot intergalactic medium - II. Ultraviolet," MNRAS 408, 1120-1138 (Oct. 2010).

[3] Bryan, G. L., "Fluids in the universe: adaptive mesh refinement in cosmology.," Comput. Sci. Eng., Vol. 1, No. 2, p. 46 - 53 1, 46-53 (Apr. 1999).

[4] O'Shea, B. W., Bryan, G., Bordner, J., Norman, M. L., Abel, T., Harkness, R., and Kritsuk, A., "Introducing Enzo, an AMR Cosmology Application," arXiv Astrophysics e-prints (Mar. 2004).

[5] Bryan, G. L., Norman, M. L., O'Shea, B. W., Abel, T., Wise, J. H., Turk, M. J., Reynolds, D. R., Collins, D. C., Wang, P., Skillman, S. W., Smith, B., Harkness, R. P., Bordner, J., Kim, J.-h., Kuhlen, M., Xu, H., Goldbaum, N., Hummels, C., Kritsuk, A. G., Tasker, E., Skory, S., Simpson, C. M., Hahn, O., Oishi, J. S., So, G. C., Zhao, F., Cen, R., Li, Y., and Enzo Collaboration, "ENZO: An Adaptive Mesh Refinement Code for Astrophysics," ApJS 211, 19 (Apr. 2014).

[6] Croft, R. A. C., Weinberg, D. H., Bolte, M., Burles, S., Hernquist, L., Katz, N., Kirkman, D., and Tytler, D., "Toward a Precise Measurement of Matter Clustering: Ly $\alpha$ Forest Data at Redshifts 2-4," ApJ 581, 20-52 (Dec. 2002).

[7] Tumlinson, J., Thom, C., Werk, J. K., Prochaska, J. X., Tripp, T. M., Katz, N., Davé, R., Oppenheimer, B. D., Meiring, J. D., Ford, A. B., O’Meara, J. M., Peeples, M. S., Sembach, K. R., and Weinberg, D. H., "The COS-Halos Survey: Rationale, Design, and a Census of Circumgalactic Neutral Hydrogen," ApJ 777, 59 (Nov. 2013).

[8] Lee, K.-G., Hennawi, J. F., Stark, C., Prochaska, J. X., White, M., Schlegel, D. J., Eilers, A.-C., Arinyo-iPrats, A., Suzuki, N., Croft, R. A. C., Caputi, K. I., Cassata, P., Ilbert, O., Garilli, B., Koekemoer, A. M., Le Brun, V., Le Fèvre, O., Maccagni, D., Nugent, P., Taniguchi, Y., Tasca, L. A. M., Tresse, L., Zamorani, G., and Zucca, E., "Ly $\alpha$ Forest Tomography from Background Galaxies: The First Megaparsec-resolution Large-scale Structure Map at z 2," ApJL 795, L12 (Nov. 2014).

[9] Tumlinson, J., Peeples, M. S., and Werk, J. K., "The Circumgalactic Medium," Annual Review of Astronomy and Astrophysics 55, 389-432 (Aug 2017).

[10] Martin, D. C., Chang, D., Matuszewski, M., Morrissey, P., Rahman, S., Moore, A., and Steidel, C. C., "Intergalactic Medium Emission Observations with the Cosmic Web Imager. I. The Circum-QSO Medium of QSO 1549+19, and Evidence for a Filamentary Gas Inflow," ApJ 786, 106 (May 2014).

\footnotetext{
${ }^{\ddagger}$ In the event that additional baffling does not address all sources of stray light, e.g. Cherenkov radiation, a redblocking filter is also an important technology demonstration for UV CCDs that address a current worry with using CCD technology over other UV detector options.
} 
[11] Martin, D. C., Chang, D., Matuszewski, M., Morrissey, P., Rahman, S., Moore, A., Steidel, C. C., and Matsuda, Y., "Intergalactic Medium Emission Observations with the Cosmic Web Imager. II. Discovery of Extended, Kinematically Linked Emission around SSA22 Ly $\alpha$ Blob 2," ApJ 786, 107 (May 2014).

[12] Martin, D. C., Matuszewski, M., O’Sullivan, D., and Hamden, E., "Evidence for Axi-symmetrical Proto-Disk formation?," Nature Astronomy (2019In Review).

[13] Borisova, E., Cantalupo, S., Lilly, S. J., Marino, R. A., Gallego, S. G., Bacon, R., Blaizot, J., Bouché, N., Brinchmann, J., Carollo, C. M., Caruana, J., Finley, H., Herenz, E. C., Richard, J., Schaye, J., Straka, L. A., Turner, M. L., Urrutia, T., Verhamme, A., and Wisotzki, L., "Ubiquitous Giant Ly $\alpha$ Nebulae around the Brightest Quasars at z

3.5 Revealed with MUSE," ApJ 831, 39 (Nov. 2016).

[14] Arrigoni Battaia, F., Hennawi, J. F., Cantalupo, S., and Prochaska, J. X., "The Stacked LY $\alpha$ Emission Profile from the Circum-Galactic Medium of z 2 Quasars," ApJ 829, 3 (Sept. 2016).

[15] Arrigoni Battaia, F., Hennawi, J. F., Prochaska, J. X., Oñorbe, J., Farina, E. P., Cantalupo, S., and Lusso, E., "QSO MUSEUM I: a sample of 61 extended Ly $\alpha$-emission nebulae surrounding z 3 quasars," MNRAS 482, 3162-3205 (Jan. 2019).

[16] O'Sullivan, D., "The FLASHES Survey I.Introduction and Preliminary Results," ApJ (2019In Preparation).

[17] Cai, Z., Hamden, E., Matuszewski, M., Prochaska, J. X., Li, Q., Cantalupo, S., Arrigoni Battaia, F., Martin, C., Neill, J. D., O'Sullivan, D., Wang, R., Moore, A., and Morrissey, P., "Keck/Palomar Cosmic Web Imagers Reveal an Enormous Ly $\alpha$ Nebula in an Extremely Overdense Quasi-stellar Object Pair Field at z $=2.45, "$ ApJL 861, L3 (July 2018).

[18] Bogosavljević, M., Lyman continuum and Lyman $\alpha$ emission from galaxies at high redshift, PhD thesis, California Institute of Technology (2010).

[19] Wisotzki, L., Bacon, R., Blaizot, J., Brinchmann, J., Herenz, E. C., Schaye, J., Bouché, N., Cantalupo, S., Contini, T., Carollo, C. M., Caruana, J., Courbot, J.-B., Emsellem, E., Kamann, S., Kerutt, J., Leclercq, F., Lilly, S. J., Patrício, V., Sandin, C., Steinmetz, M., Straka, L. A., Urrutia, T., Verhamme, A., Weilbacher, P. M., and Wendt, M., "Extended Lyman $\alpha$ haloes around individual high-redshift galaxies revealed by MUSE," A\&A 587, A98 (Mar. 2016).

[20] Steidel, C. C., Bogosavljević, M., Shapley, A. E., Kollmeier, J. A., Reddy, N. A., Erb, D. K., and Pettini, M., "Diffuse Ly $\alpha$ Emitting Halos: A Generic Property of High-redshift Star-forming Galaxies," ApJ 736, 160 (Aug. 2011).

[21] Matuszewski, M., Chang, D., Crabill, R. M., Martin, D. C., Moore, A. M., Morrissey, P., and Rahman, S., "The Cosmic Web Imager: an integral field spectrograph for the Hale Telescope at Palomar Observatory: instrument design and first results," in [Society of Photo-Optical Instrumentation Engineers (SPIE) Conference Series], Society of Photo-Optical Instrumentation Engineers (SPIE) Conference Series 7735, 0 (July 2010).

[22] Tuttle, S. E., Schiminovich, D., Milliard, B., Grange, R., Martin, D. C., Rahman, S., Deharveng, J., McLean, R., Tajiri, G., and Matuszewski, M., "The FIREBall fiber-fed UV spectrograph," in [Society of Photo-Optical Instrumentation Engineers (SPIE) Conference Series], Presented at the Society of PhotoOptical Instrumentation Engineers (SPIE) Conference 7014 (Aug. 2008).

[23] Melso, N., Schiminovich, D., Matuszewski, M., Montel, J., Pirot, E., Gross, J., Zorilla, J., Ong, H. R., Lemon, M., Smiley, B., Hamden, E. T., Picouet, V., Vibert, D., O’Sullivan, D., Kyne, G., Martin, D. C., Milliard, B., Grange, R., Nikzad, S., Evrard, J., Hoadley, K., Lingner, N., Balard, P., Crabill, M., Pascal, S., Mirc, F., Bray, N., Jewell, A., Augustin, R., Gomes, A., Tapie, P., Soors, X., Zerone, I., and Saccoccio, M., "FIREBall-2 Field Acquisition and Fine Guiding System: In-flight Performance and Scientific Implications," JATIS (2019In Preparation).

[24] Grange, R., Lemaitre, G. R., Quiret, S., Milliard, B., Pascal, S., and Origné, A., "Multi object spectrograph of the Fireball balloon experiment," in [Society of Photo-Optical Instrumentation Engineers (SPIE) Conference Series], Society of Photo-Optical Instrumentation Engineers (SPIE) Conference Series 9144, 30 (July 2014). 
[25] Hamden, E. T., Jewell, A. D., Shapiro, C. A., Cheng, S. R., Goodsall, T. M., Hennessy, J., Hoenk, M., Jones, T., Gordon, S., Ong, H. R., Schiminovich, D., Martin, D. C., and Nikzad, S., "Charge-coupled devices detectors with high quantum efficiency at UV wavelengths," Journal of Astronomical Telescopes, Instruments, and Systems 2, 036003 (July 2016).

[26] Nikzad, S., Jewell, A. D., Hoenk, M. E., Jones, T. J., Hennessy, J., Goodsall, T., Carver, A. G., Shapiro, C., Cheng, S. R., Hamden, E. T., Kyne, G., Martin, D. C., Schiminovich, D., Scowen, P., France, K., McCandliss, S., and Lupu, R. E., "High-efficiency UV/optical/NIR detectors for large aperture telescopes and UV explorer missions: development of and field observations with delta-doped arrays," Journal of Astronomical Telescopes, Instruments, and Systems 3, 036002 (July 2017).

[27] Kyne, G., Hamden, E. T., Martin, D. C., Schiminovich, D., Milliard, B., Grange, R., Nikzad, S., Evrard, J., Montel, J., Pirot, E., Hoadley, K., O’Sullivan, D., Matuszewski, M., Lingner, N., Melso, N., Picouet, V., Vibert, D., Balard, P., Crabill, M., Pascal, S., Gross, J., Zorilla, J., Ong, H. R., Mirc, F., Bray, N., Jewell, A., Augustin, R., Smiley, B., Lemon, M., Gomes, A., Tapie, P., Soors, X., Zerone, I., and Saccoccio, M., "Electron Multiplying CCDs for FIREBall-2," JAI (2019In Preparation).

[28] Montel, J. and al., "FIREBALL-2 (2018) in flight pointing performances," (2019).

[29] Ardila, D. R., Shkolnik, E., Scowen, P., Jewell, A., Nikzad, S., Bowman, J., Fitzgerald, M., Jacobs, D., Spittler, C., Barman, T., Peackock, S., Beasley, M., Gorgian, V., Llama, J., Meadows, V., Swain, M., and Zellem, R., "The Star-Planet Activity Research CubeSat (SPARCS): A Mission to Understand the Impact of Stars in Exoplanets," arXiv e-prints , arXiv:1808.09954 (Aug 2018). 